WM-98-118

June 12, 2018

\title{
Vacuum Stability Bounds in the Two-Higgs Doublet Model
}

\author{
Shuquan Nie and Marc Sher \\ Nuclear and Particle Theory Group \\ Physics Department \\ College of William and Mary, Williamsburg, VA 23187, USA
}

\begin{abstract}
In the standard model, the requirements of vacuum stability and the validity of perturbation theory up to the unification scale force the mass of the Higgs boson to be approximately between $130 \mathrm{GeV}$ and $180 \mathrm{GeV}$. We re-examine these requirements in the (non-supersymmetric) two-Higgs doublet model, in the light of the large top quark mass, and constrain the masses of the Higgs bosons in this model. It is found that the mass of the charged Higgs boson must be lighter than $150 \mathrm{GeV}$. This bound is below the lower bound in the popular model-II two-Higgs doublet model, and thus we conclude that this model cannot be valid up to the unification scale. The bounds on the neutral Higgs scalars are also discussed.
\end{abstract}


The mass of the Higgs boson in the standard model is, at first sight, completely arbitrary. It depends on the scalar self-coupling, $\lambda$, which is a free parameter. However, rather stringent bounds on the mass can be obtained[1], 2] by requiring that (a) $\lambda$ remain perturbative up to a large scale (generally taken to be the unification scale of approximately $10^{16} \mathrm{GeV}$-the precise value doesn't much matter), and (b) that the vacuum of the standard model remain stable up to that large scale. The first condition gives an upper bound to the Higgs mass of approximately $180 \mathrm{GeV}$. The second condition is virtually identical to requiring that $\lambda$ remain positive up to the large scale, and that gives a lower bound to the Higgs mass of approximately $130 \mathrm{GeV}$. Thus, these two conditions strongly constrain the mass of the Higgs boson to be between 130 and $180 \mathrm{GeV}$.

It is easy to see where these constraints arise. The renormalization group equation for the scalar self-coupling is of the form $\frac{d \lambda}{d t}=a \lambda^{2}-b g_{Y}^{4}$, where $g_{Y}$ is the top quark Yukawa coupling. If $\lambda$ is large, the first term dominates, and $\lambda$ blows up; if it is small, the second term dominates, and $\lambda$ becomes negative, leading to a vacuum instability. Only for $\lambda$ near the fixed point of this equation does $\lambda$ remain positive and finite from the electroweak to unification scale.

The most straightforward extension of the standard model is the two-Higgs doublet model(2HDM). It includes two complex scalar doublets (see Ref. [1] for a review),

$$
\Phi_{1}=\left(\begin{array}{c}
\chi_{1}^{+} \\
\left(\phi_{1}+i \chi_{1}\right) / \sqrt{2}
\end{array}\right), \quad \Phi_{2}=\left(\begin{array}{c}
\chi_{2}^{+} \\
\left(\phi_{2}+i \chi_{2}\right) / \sqrt{2}
\end{array}\right)
$$

Of the eight real fields, three must become the longitudinal components of the $W^{ \pm}$and $\mathrm{Z}$ bosons after the spontaneous symmetry breaking. Linear combinations of $\chi_{1}^{ \pm}$and $\chi_{2}^{ \pm}$ are absorbed into the longitudinal parts of the $W^{ \pm}$bosons and a linear combination of $\chi_{1}$ and $\chi_{2}$ give mass to the $\mathrm{Z}$ boson. Five physical Higgs scalars will remain: a charged scalar $\chi^{ \pm}$and three neutral scalars $\phi_{1}, \phi_{2}$ and the other linear combination of $\chi_{1}$ and $\chi_{2}$, 
called $\chi^{0}$.

Given how stringently the Higgs mass in the standard model is constrained, one might ask how stringently the masses of the scalars in the two-doublet model are constrained. There are many more self-couplings (which could potentially diverge by the unification scale) and more directions in field space where an instability could arise. In this paper, we examine these constraints in the two-doublet model. This is not new- the constraints have been examined before 1 , 3], but the top quark mass was unknown at the time (and only values up to about $130 \mathrm{GeV}$ for the top quark mass were considered).

A potential danger with additional Higgs doublets is the possibility of flavor-changing neutral currents(FCNC). It is well known that FCNC are highly suppressed relative to the charged current processes, so it would be desirable to "naturally" suppress them in these models. If all quarks with the same quantum numbers couple to the same scalar multiplet, then FCNC will be absent. This led Glashow and Weinberg[国 to propose a discrete symmetry which force all the quarks of a given charge to couple to only one doublet. There are two such possible discrete symmetries in the 2HDM,

$$
(I) \phi_{2} \rightarrow-\phi_{2} \quad(I I) \phi_{2} \rightarrow-\phi_{2}, d_{R}^{i} \rightarrow-d_{R}^{i}
$$

In model I, all quarks couple to the same doublet, and no quarks couple to the other doublet. In model II, the $\mathrm{Q}=2 / 3$ quarks couple to one doublet and the $\mathrm{Q}=-1 / 3$ quarks couple to the other doublet.

The most general potential subject to one of the discrete symmetries, for two doublets of hypercharge +1 (if one of the doublets has hypercharge -1 , our arguments will be unaffected), is

$$
\begin{aligned}
V & =\mu_{1}^{2} \Phi_{1}^{+} \Phi_{1}+\mu_{2}^{2} \Phi_{2}^{+} \Phi_{2}+\lambda_{1}\left(\Phi_{1}^{+} \Phi_{1}\right)^{2}+\lambda_{2}\left(\Phi_{2}^{+} \Phi_{2}\right)^{2} \\
& +\lambda_{3}\left(\Phi_{1}^{+} \Phi_{1}\right)\left(\Phi_{2}^{+} \Phi_{2}\right)+\lambda_{4}\left(\Phi_{1}^{+} \Phi_{2}\right)^{2}+\frac{1}{2} \lambda_{5}\left[\left(\Phi_{1}^{+} \Phi_{2}\right)^{2}+\left(\Phi_{2}^{+} \Phi_{1}\right)^{2}\right]
\end{aligned}
$$


the vacuum expectation values of $\Phi_{1}$ and $\Phi_{2}$ can be written as

$$
\Phi_{1}=1 / \sqrt{2}\left(\begin{array}{c}
0 \\
v_{1}
\end{array}\right), \quad \Phi_{2}=1 / \sqrt{2}\left(\begin{array}{c}
0 \\
v_{2}
\end{array}\right)
$$

with $v_{1}^{2}+v_{2}^{2}=\sqrt{2} G_{F}=(247 G e V)^{2}$. The masses of the physical scalars are given by

$$
\begin{array}{cc}
m_{\chi^{ \pm}}^{2}=-\frac{1}{2}\left(\lambda_{4}+\lambda_{5}\right)\left(v_{1}^{2}+v_{2}^{2}\right), & m_{\chi^{0}}^{2}=-\lambda_{5}\left(v_{1}^{2}+v_{2}^{2}\right), \\
m_{\phi}^{2}=\frac{1}{2}\left(A+B+\sqrt{(A-B)^{2}+4 C^{2}}\right), & m_{\eta}^{2}=\frac{1}{2}\left(A+B-\sqrt{(A-B)^{2}+4 C^{2}}\right),
\end{array}
$$

where $A=2 \lambda_{1} v_{1}^{2}, B=2 \lambda_{2} v_{2}^{2}$ and $C=\left(\lambda_{3}+\lambda_{4}+\lambda_{5}\right) v_{1} v_{2}$.

It is required that all scalar boson masses-squared must be positive. This implies that

$$
\begin{aligned}
& \text { (i) } \lambda_{5}<0 \\
& \text { (ii) } \lambda_{4}+\lambda_{5}<0 \\
& \text { (iii) } \lambda_{1}>0 \\
& \text { (iv) } \lambda_{2}>0 \\
& \text { (v) } 2 \sqrt{\lambda_{1} \lambda_{2}}>\lambda_{3}+\lambda_{4}+\lambda_{5}
\end{aligned}
$$

In the standard model the positivity of the scalar boson mass-squared implies that $\lambda>0$, To ensure vacuum stability for all scales up to $M_{X}$, one must have $\lambda\left(q^{2}\right)>0$ for all $q^{2}$ from $M_{Z}^{2}$ to $M_{X}^{2}$. Similarly, to ensure vacuum stability in the $2 \mathrm{HDM}$ up to $M_{X}^{2}$, one must require that all of the five constraints be valid up to $M_{X}^{2}$. If the condition $\lambda_{1}$ or $\lambda_{2}>0$ is violated, the potential will be unstable in the $\phi_{1}$ or $\phi_{2}$ direction. if the condition $(v)$ is violated, the potential will be unstable in some dirction in $\phi_{1}-\phi_{2}$ plane. If the condition $\lambda_{4}+\lambda_{5}<0$ is violated, a new minimum which breaks charge will be formed. And if $\lambda_{5}$ become positive, a new minimum which violates $\mathrm{CP}$ will be formed. Thus it is required that all of the constraints should be valid up to $M_{X}$. At the same time it is physically reasonable to demand that all $\lambda$ 's be finite (or perturbative) up to $M_{X}$.

Starting with $\lambda_{1}, \lambda_{2}, \lambda_{3}, \lambda_{4}, \lambda_{5}$ and $\tan \beta$ at the electroweak scale, the renormalization group equations are integrated numerically to check whether one of the five constraints is violated or whether any of the couplings become nonperturbative before reaching $M_{X}$. 
The results give an allowed region in the six-dimensional parameter space. To explore this region, we choose the six parameters to be the four physical scalar masses, $\tan \beta$ and $\lambda_{3}$. For a point in this parameter-space to be acceptable, all of the above constraints, as well as perturbativity, must be satisfied at all scales up to $M_{X}$. In practice, since $\lambda_{3}$ is unmeasureable, we consider the other five parameters as starting points, and see if any initial values of $\lambda_{3}$ give acceptable values. In this way, we determine if a given point in the five-dimensional space of the scalar masses and $\tan \beta$ is acceptable.

It is, of course, difficult to plot a region in five-dimensional space. However, the basic features can easily be seen with a few examples. Let us first consider the case in which $m_{\chi^{ \pm}}=m_{\chi^{o}}=100 \mathrm{GeV}$. We choose $\tan \beta=2$, and plot the allowed region in the neutral scalar mass plane. The region is shown in Figure 1. For $m_{\eta}$ between 40 and $88 \mathrm{GeV}$, one sees that the value of $m_{\phi}$ must lie below $180 \mathrm{GeV}$ and above a value which ranges from $130 \mathrm{GeV}$ to $100 \mathrm{GeV}$; this bound is very similar to the result in the standard model. However, there are no solutions in which $m_{\eta}$ is greater than $88 \mathrm{GeV}$ or below $40 \mathrm{GeV}$. Below the region, $\lambda_{1}$ becomes negative, above the region $\lambda_{1}$ becomes non-perturbative, to the left and to the right of the region, the constraint $(v)$ is violated.

One can now vary some of the three input parameters to see how this region changes. As the charged Higgs mass increases, the region shrinks dramatically, disappearing when it reaches $140 \mathrm{GeV}$, as shown in Figure 2. As $\tan \beta$ increases, the region shifts to smaller values of $m_{\eta}$, as shown in Figure 3. This is not surprising since $m_{\eta}$ becomes small as $\tan \beta \rightarrow \infty$. As $\tan \beta$ decreases, the size of the allowed region shrinks, since the top quark Yukawa coupling is getting larger, leading to an instability. Finally, varying $m_{\chi^{o}}$ gives the result in Figure 4. As in the charged Higgs case, the allowed region disappears when the pseudoscalar mass exceeds $140 \mathrm{GeV}$.

The most important result is seen from Figure 2, where this is a stringent upper bound on the charged Higgs mass. By optimizing $\tan \beta$ and $m_{\chi^{o}}$, we find that the maximum 
allowed value for the charged Higgs mass is $150 \mathrm{GeV}$.

What are the experimental constraints? As discussed in Ref. [5], there are very few constraints on the neutral scalar masses. If one takes the $\chi^{o}$ mass to be $100 \mathrm{GeV}$, the only constraints come from the Bjorken process, $e^{+} e^{-} \rightarrow Z^{*} \rightarrow Z \eta$, and the rate can be significantly reduced by judicious choice of the mixing angle. So no bounds on $m_{\eta}$ are relevant. There is, however, a strong bound [5, [6] on $m_{\chi}^{ \pm}$coming from $B \rightarrow X_{s} \gamma$. In Model II, this process forces the charged Higgs mass to be greater than $165 \mathrm{GeV}$. This in inconsistent with our upper bound. Model I, however, has no such constraint, and the charged Higgs mass could be as light as $45 \mathrm{GeV}$.

We conclude that the popular two-Higgs doublet model, Model II, can not be valid up to the unification scale. Model I is not excluded, however we do find that the charged Higgs mass must be lighter than $150 \mathrm{GeV}$, the lightest neutral scalar must be lighter than $110 \mathrm{GeV}$ and the pseudoscalar must be lighter than $140 \mathrm{GeV}$ for the model to be valid up to the unification scale.

\section{References}

[1] M. Sher, Physics Reports 179 (1989) 273

[2] M. Sher, Phys. Lett. B317 (1993) 159 ; J.A. Casas, J.R. Espinosa and M. Quiros, Phys. Lett. B324 (1995) 171 and Phys. Lett. B382 (1996) 374 ; J.R. Espinosa and M. Quiros, Phys. Lett. B353 (1995) 257 .

[3] M. Sher, Perspectives in Higgs Physics I, G. Kane, ed. (World Scientific, 1994).

[4] S.L. Glashow and S. Weinberg, Phys. Rev. D15 (1977) 1958 .

[5] F.M. Brozumati and C. Grueb, hep-ph/9810240, Proc. of XXIX Int'l Conference on High Enegy Physics, Vancouver, BC, Canada, July 1998. 
[6] T.M. Aliev and E.O. Iltan, hep-ph/9803272; F.M. Borzumati and A. Djouadi, hepph/9806301. 
Figure 1: The allowed region in the neutral scalar mass plane, with $m_{\chi^{ \pm}}=m_{\chi^{o}}=100$ $\mathrm{GeV}$ and $\tan \beta=2$.

Figure 2: The allowed region in the neutral scalar mass plane for various values of the charged Higgs mass (in GeV), with the $\chi^{o}$ mass chosen to be $100 \mathrm{GeV}$ and $\tan \beta=2$.

Figure 3: The allowed region in the neutral scalar mass plane for various values of $\tan \beta$, with the $\chi^{o}$ and $\chi^{ \pm}$masses chosen to be $100 \mathrm{GeV}$.

Figure 4: The allowed region in the neutral scalar mass plane for various values of the pseudoscalar Higgs mass (in $\mathrm{GeV}$ ), with the $\chi^{ \pm}$mass chosen to be $100 \mathrm{GeV}$ and $\tan \beta=2$. 


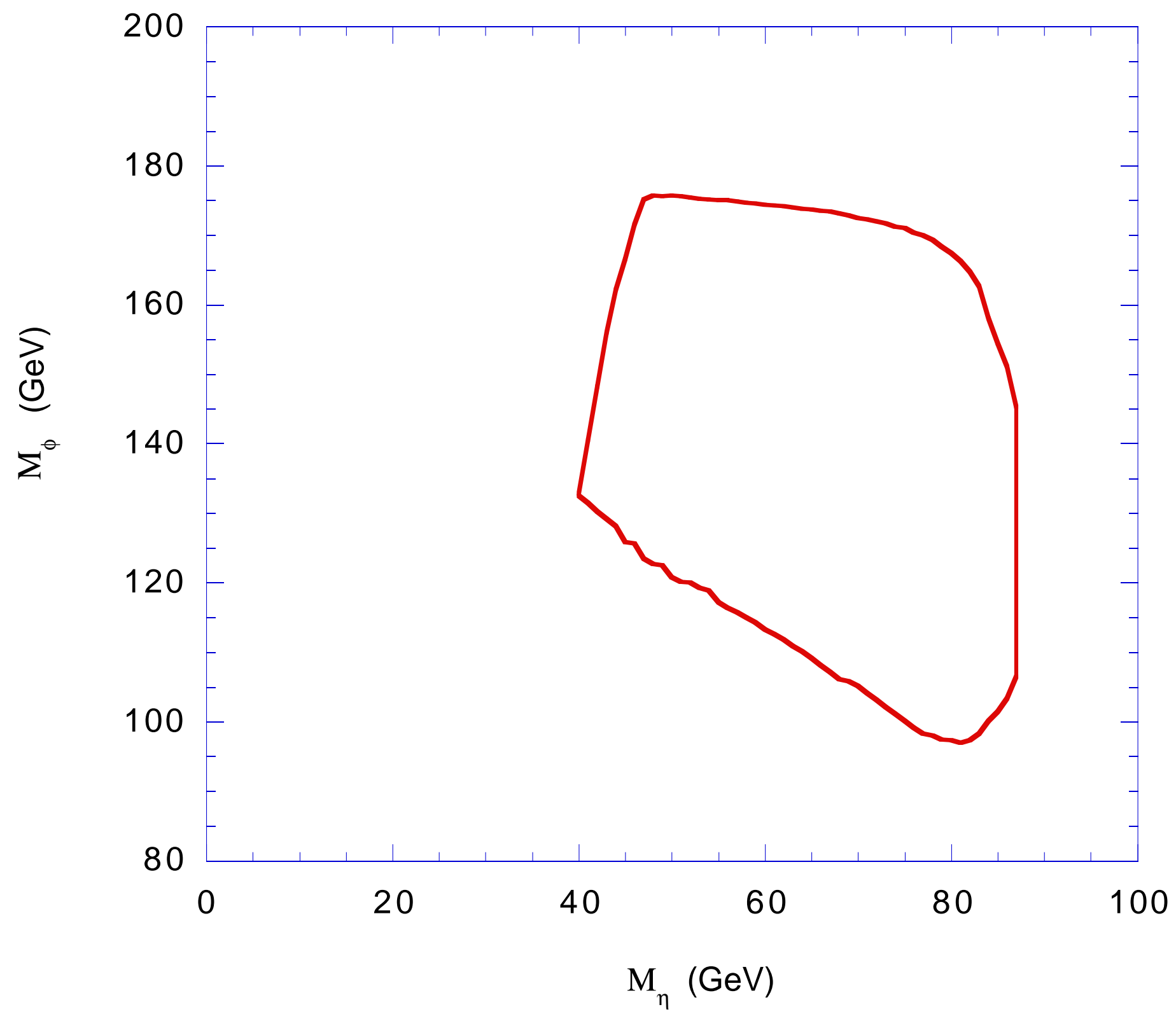




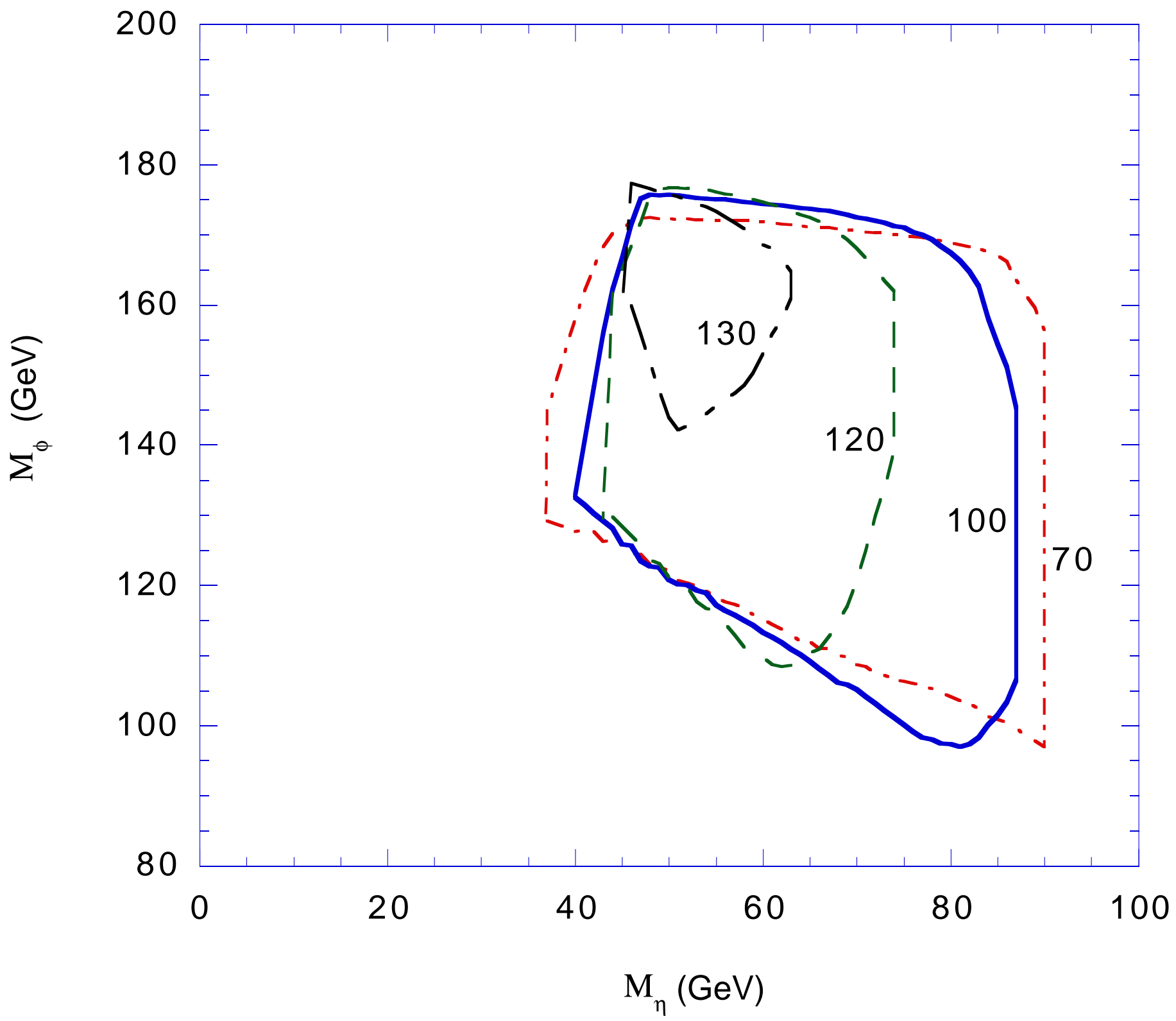




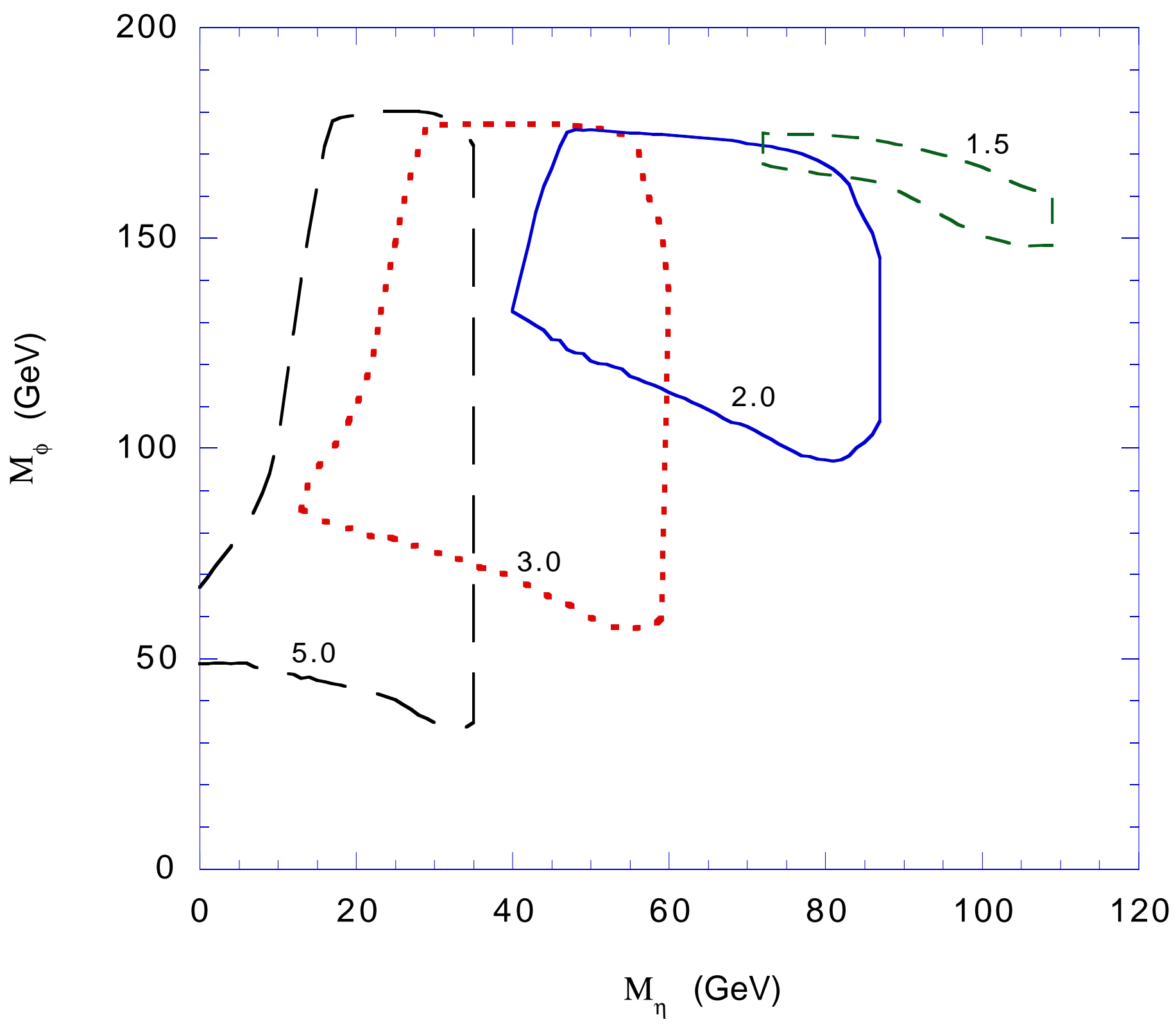




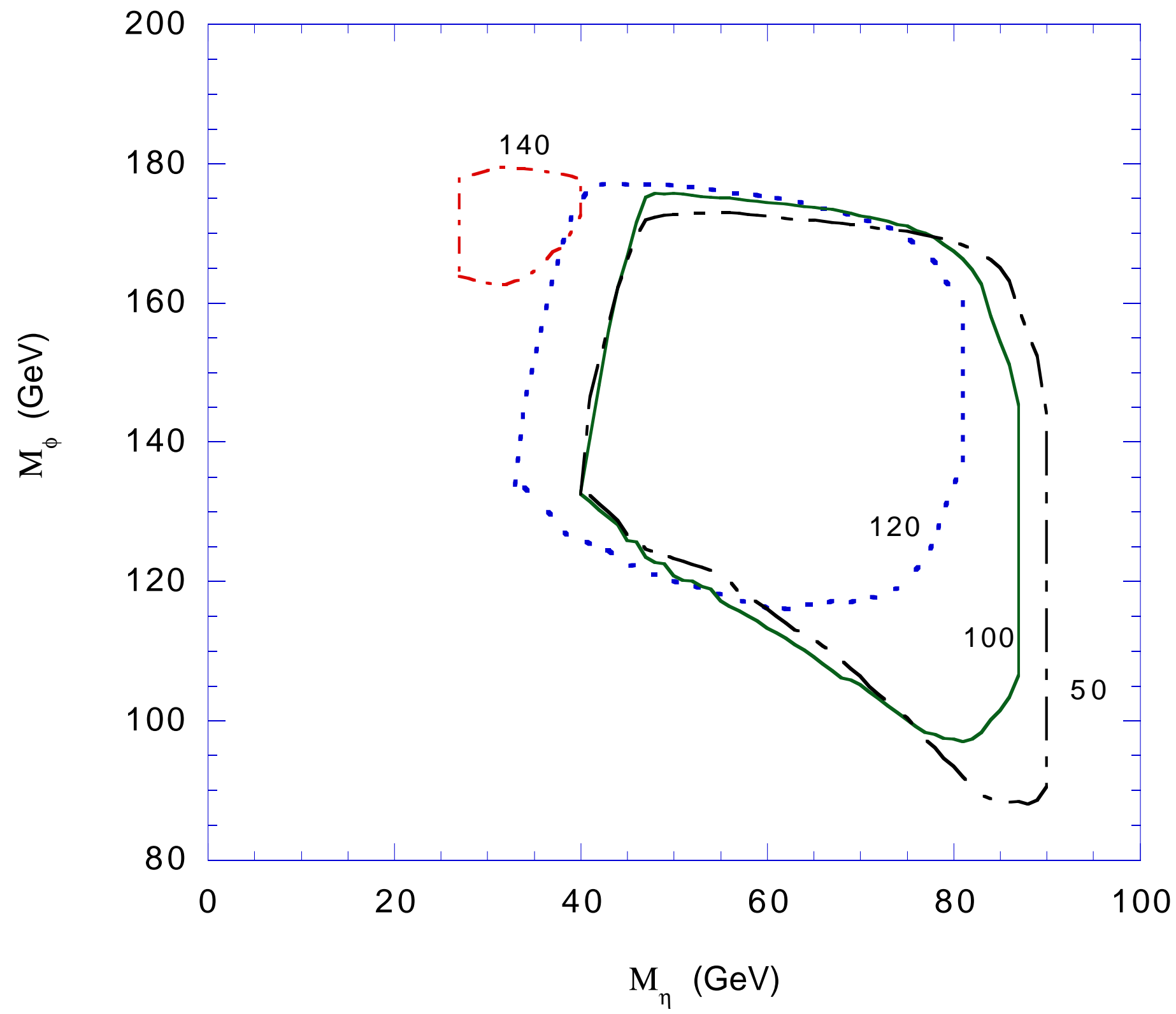

